\title{
Fitossociologia e síndrome de dispersão em um trecho de floresta atlântica, em Paulista - PE
}

\author{
Raquel Elvira Cola ${ }^{*}$, Sthéfany Carolina de Melo Nobre ${ }^{1}$, Débora dos Santos Farias ${ }^{1}$, Lucas Galdino da \\ Silva ${ }^{1}$, Andréa de Vasconcelos Freitas Pinto $^{2}$, Carlos Frederico Lins e Silva Brandão ${ }^{3}$
}

\begin{abstract}
RESUMO: O objetivo foi realizar o levantamento fitossociológico com síndrome de dispersão do componente arbóreo adulto em um trecho de floresta ombrófila densa, localizado em Paulista - Pernambuco. O trecho possui 23,7 ha. Foram lançadas parcelas aleatórias de $200 \mathrm{~m}^{2}$, somando 20 parcelas com área total de 0,4 ha. O nível de inclusão dos indivíduos arbóreos foi com circunferência a altura do peito (CAP) igual ou maior que $15 \mathrm{~cm}$. Todos os indivíduos mensurados foram identificados botanicamente seguindo a classificação APG III. A caracterização fitossociológica foi feita através dos seguintes parâmetros: densidade absoluta e relativa, dominância absoluta e relativa, frequência absoluta e relativa e valor de importância. O Índice de Diversidade de Shannon (H') também foi calculado. Para a síndrome de dispersão, as espécies foram classificadas como zoocóricas ou dispersadas por elementos abióticos. As espécies com maior valor de importância foram Tapirira guianensis, Schefflera morototoni, Pogonophora schomburgkiana, Cupania racemosa, e Miconia prasina, respectivamente. O Índice de Shannon foi de 3,44. A síndrome de dispersão demonstrou que $80,9 \%$ das espécies são dispersadas por zoocoria e 14,3\% por mecanismos abióticos. O componente arbóreo adulto se encontra dentro dos padrões de riqueza e abundância encontrados em remanescentes florestais de Pernambuco.
\end{abstract}

Palavras-chave: fragmentação, floresta ombrófila densa, índice de diversidade, componente arbóreo.

\section{Phytosociology and dispersion syndrome of the Mata do Ronca, in Paulista - PE}

\begin{abstract}
The objective of was to conduct a phytosociological survey with the dispersion syndrome of the adult arboreal component in a stretch of ombrophilous dense forest, located in Paulista - Pernambuco. The stretch has 23.7 ha. Random plots of $200 \mathrm{~m}^{2}$ were added, adding 20 plots with a total area of 0.4 ha. The inclusion level of the arboreal individuals was with chest circumference (CAP) equal to or greater than $15 \mathrm{~cm}$. All individuals measured were classified botanically following the APG III classification. The phytosociological characterization was made through the following parameters: absolute and relative dominance, absolute and relative frequency and value of importance. The Shannon Diversity Index $\left(H^{\prime}\right)$ was also calculated. For the dispersion syndrome, the species were classified as zoochoric or dispersed by abiotic elements. The species with the highest value of importance were Tapirira guianensis, Schefflera morototoni, Pogonophora schomburgkiana, Cupania racemosa, and Miconia prasina, respectively. The Shannon Index was 3.44. The analysis of the dispersion syndrome showed that $80.9 \%$ of the species are dispersed by zoochoric and $14.3 \%$ by abiotic elements. The adult tree component is within the patterns of richness and abundance found in forest remnants of Pernambuco.
\end{abstract}

Keywords: fragmentation, ombrophilous dense forest, diversity index, adult tree component.

\section{INTRODUÇÃO}

O levantamento fitossociológico, em florestas nativas, tem como objetivo inferir acerca de parâmetros como: densidade, dominância, índice de valor de importância, estrutura, riqueza, posição ecológica, índice de regeneração natural, etc. (FREITAS, MAGALHÃES, 2012). Além disso, esse estudo envolve o conhecimento das interrelações de espécies vegetais da comunidade vegetal em questão. As informações qualitativas tornam-se quantitativas e determinam qual o nível de desenvolvimento da área.

Uma das formas de auxiliar os levantamentos fitossociológicos é através na análise da síndrome de dispersão de sementes de uma determinada área. Esse processo representa uma importante etapa no ciclo reprodutivo das plantas. Já que representa um importante mecanismo para a sobrevivência das plântulas, além de participar da colonização de novos ambientes e da dispersão para micro-habitats favoráveis (PEDRONI, 1993; BUSTAMANTE, CANALS 1995; HOWE, SMALLWOOD, 1982).

Neste contexto conhecer a estrutura e dinâmica de uma floresta, termina contribuindo e gerando subsídios para a conservação dos recursos naturais, bem como para a conservação de áreas similares (CHAVES et al., 2013; BULHÕES et al., 2015).

Desta forma o objetivo deste trabalho foi de realizar o levantamento fitossociológico com a síndrome de dispersão do componente arbóreo adulto em um trecho de floresta atlântica, localizado no município de Paulista - Pernambuco.

\footnotetext{
Recebido em 05/10/2019, Aceito para publicação em 15/10/2019

${ }^{1}$ Universidade Federal de Alagoas

*E-mail: raquelelvira@outlook.com
} 


\section{MATERIAL E MÉTODOS}

A área estudada está localizada no município de Paulista, no estado do Pernambuco. O trecho faz parte da Mata do Ronca (141 ha) e possui 23,7 ha.

Foram lançadas parcelas aleatórias de $200 \mathrm{~m}^{2}(10$ x $20 \mathrm{~m}$ ), somando 20 parcelas com área total de $4.000 \mathrm{~m}^{2}$ ou 0,4 ha (Figura 1). As parcelas foram georrefereciadas com o auxílio do GPS Gramin map $76 \mathrm{Cx}$.

O nível de inclusão considerado dos indivíduos arbóreos foi com circunferência a altura do peito (CAP) igual ou maior que $15 \mathrm{~cm} \mathrm{e,} \mathrm{a} \mathrm{partir} \mathrm{disso,}$ foram medidos o CAP e a altura de cada um. Todos os indivíduos mensurados foram identificados botanicamente seguindo a classificação APG III.

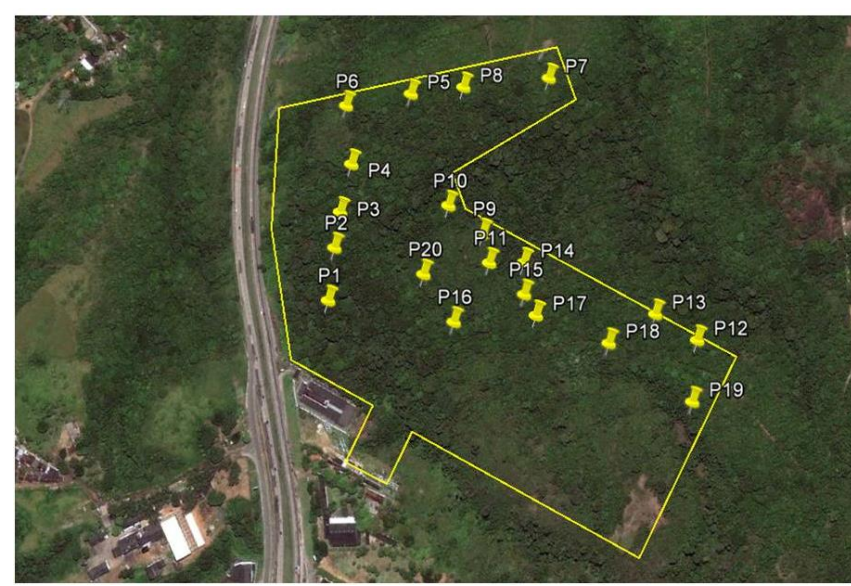

Figura 1 - Localização das parcelas utilizadas no levantamento fitossociológico em um trecho estudado na Mata do Ronca, Paulista - PE.

Seguindo a metodologia de Martins (1991), a caracterização fitossociológica é feita através dos seguintes parâmetros fitossociológicos: densidade absoluta e relativa, dominância absoluta e relativa, frequência absoluta e relativa e valor de importância.
O Índice de Diversidade de Shannon (H') foi utilizado para analisar a diversidade florística.

A síndrome de dispersão seguiu a classificação determinada por Van der Pijl (1982), onde as espécies foram classificadas em zoocóricas (dispersas por animais) e por elementos abióticos (como vento, gravidade e água). Para obter as informações por espécie, foi pesquisado em livros, teses e artigos científicos.

\section{RESULTADOS E DISCUSSÃO}

Foram amostrados um total de 558 indivíduos em uma área de $4.000 \mathrm{~m}^{2}$, totalizando uma densidade absoluta de 1.395 indivíduos/ha. Os indivíduos estão distribuídos em 63 espécies arbóreas, sendo 49 identificados em nível de espécie, 8 até o gênero, 4 até a família botânica e 2 não identificadas (NI 1 e 2).

De acordo com as informações da Tabela 1, as espécies com maior densidade foram Tapirira guianensis (187,5 ind./ha), Schefflera morototoni (170 ind./ha), Pogonophora schomburgkiana (90 ind./ha), Miconia prasina (70 ind./ha) e Thyrsodium spruceanum (67,5 ind./ha), representando $41,9 \%$ do total de indivíduos por hectare.

As espécies Tapirira guianensis $\left(2,42 \mathrm{~m}^{2} / \mathrm{ha}\right)$, Schefflera morototoni $\left(1,07 \mathrm{~m}^{2} / \mathrm{ha}\right)$, Parkia pendula $\left(0,61 \mathrm{~m}^{2} / \mathrm{ha}\right)$, Cupania racemosa $\left(0,60 \mathrm{~m}^{2} / \mathrm{ha}\right)$ e Abarema sp. $\left(0,54 \mathrm{~m}^{2} / \mathrm{ha}\right)$ representaram $46,8 \%$ do total de área basal calculado no fragmento. Mesmo a espécie Parkia pendula tendo a menor abundância, sua inclusão nesse grupo foi devido ao grande porte de um único indivíduo dessa espécie, com $48,7 \mathrm{~cm}$.

Tabela 1. Levantamento fitossociológico em um fragmento de Floresta Atlântica no município de Paulista - PE.

\begin{tabular}{|c|c|c|c|c|c|c|c|c|}
\hline Nome científico & $\mathrm{N}$ & DA & DR & DoA & DoR & FA & FR & VI \\
\hline Tapirira guianensis Aubl. & 75 & 187,5 & 13,44 & 2,42 & 21,50 & 100 & 7,1 & 42,1 \\
\hline $\begin{array}{l}\text { Schefflera morototoni (Aubl.) et al. } \\
\text { Pogonophora schomburgkiana Miers }\end{array}$ & 68 & 170,0 & 12,19 & 1,07 & 9,55 & 90 & 6,4 & 28,1 \\
\hline ex Benth. & 36 & 90,0 & 6,45 & 0,37 & 3,33 & 75 & 5,3 & 15,1 \\
\hline Cupania racemosa (Vell.) Radlk. & 23 & 57,5 & 4,12 & 0,60 & 5,30 & 55 & 3,9 & 13,3 \\
\hline Miconia prasina (Sw.) DC. & 28 & 70,0 & 5,02 & 0,31 & 2,78 & 35 & 2,5 & 10,3 \\
\hline Inga capitata Desv. & 21 & 52,5 & 3,76 & 0,35 & 3,09 & 45 & 3,2 & 10,1 \\
\hline Abarema sp. & 11 & 27,5 & 1,97 & 0,54 & 4,81 & 45 & 3,2 & 10,0 \\
\hline $\begin{array}{l}\text { Thyrsodium spruceanum Benth. } \\
\text { Eschweilera ovata (Cambess.) Mart. }\end{array}$ & 27 & 67,5 & 4,84 & 0,19 & 1,71 & 45 & 3,2 & 9,7 \\
\hline ex Miers. & 22 & 55,0 & 3,94 & 0,16 & 1,41 & 60 & 4,3 & 9,6 \\
\hline $\begin{array}{l}\text { Guatteria pogonopus Mart. } \\
\text { Parkia pendula (Willd.) Benth. ex }\end{array}$ & 16 & 40,0 & 2,87 & 0,23 & 2,06 & 55 & 3,9 & 8,8 \\
\hline Walp. & 7 & 17,5 & 1,25 & 0,61 & 5,40 & 25 & 1,8 & 8,4 \\
\hline Cecropia sp. & 17 & 42,5 & 3,05 & 0,36 & 3,22 & 30 & 2,1 & 8,4 \\
\hline Byrsonima sericea DC. & 15 & 37,5 & 2,69 & 0,36 & 3,22 & 30 & 2,1 & 8,0 \\
\hline
\end{tabular}




\begin{tabular}{|c|c|c|c|c|c|c|c|c|}
\hline Alseis pickelii Pilg. \& Schmale. & 11 & 27,5 & 1,97 & 0,13 & 1,20 & 45 & 3,2 & 6,4 \\
\hline Casearia javitensis Kunth. & 14 & 35,0 & 2,51 & 0,13 & 1,18 & 30 & 2,1 & 5,8 \\
\hline Sclerolobium densiflorum Benth. & 10 & 25,0 & 1,79 & 0,10 & 0,92 & 35 & 2,5 & 5,2 \\
\hline Vismia guianensis (Aubl.) Choisy. & 10 & 25,0 & 1,79 & 0,08 & 0,73 & 30 & 2,1 & 4,7 \\
\hline $\begin{array}{l}\text { Albizia pedicellaris (DC.) L. Rico. } \\
\text { Maytenus disthicophylla Mart. ex }\end{array}$ & 6 & 15,0 & 1,08 & 0,15 & 1,33 & 25 & 1,8 & 4,2 \\
\hline & 7 & 17,5 & 1,25 & 0,09 & 0,76 & 30 & 2,1 & 4,2 \\
\hline Woodson. & 9 & 22,5 & 1,61 & 0,07 & 0,63 & 25 & 1,8 & 4,0 \\
\hline $\begin{array}{l}\text { Miconia minutiflora (Bonpl.) DC. } \\
\text { Ouratea hexasperma (A. St.-Hill.) - }\end{array}$ & 11 & 27,5 & 1,97 & 0,06 & 0,57 & 20 & 1,4 & 4,0 \\
\hline Baill. & 6 & 15,0 & 1,08 & 0,07 & 0,63 & 30 & 2,1 & 3,8 \\
\hline Pouteria sp. & 5 & 12,5 & 0,90 & 0,16 & 1,43 & 15 & 1,1 & 3,4 \\
\hline Xylopia frutescens Aubl. & 6 & 15,0 & 1,08 & 0,13 & 1,17 & 15 & 1,1 & 3,3 \\
\hline Aspidosperma discolor A. DC. & 1 & 2,5 & 0,18 & 0,30 & 2,68 & 5 & 0,4 & 3,2 \\
\hline Simarouba amara Aubl. & 4 & 10,0 & 0,72 & 0,15 & 1,34 & 15 & 1,1 & 3,1 \\
\hline Guatteria sp. & 5 & 12,5 & 0,90 & 0,05 & 0,43 & 25 & 1,8 & 3,1 \\
\hline Henriettea succosa (Aubl.) DC. & 4 & 10,0 & 0,72 & 0,07 & 0,66 & 20 & 1,4 & 2,8 \\
\hline Virola gardineri (A.DC.) Warb. & 2 & 5,0 & 0,36 & 0,18 & 1,63 & 10 & 0,7 & 2,7 \\
\hline Myrcia silvatica (G. Mey.) DC. & 5 & 12,5 & 0,90 & 0,03 & 0,25 & 20 & 1,4 & 2,6 \\
\hline $\begin{array}{l}\text { Spondias mombin } \mathrm{L} \text {. } \\
\text { Pouteria peduncularis Mart. \& }\end{array}$ & 1 & 2,5 & 0,18 & 0,23 & 2,03 & 5 & 0,4 & 2,6 \\
\hline Eichler ex Miq. & 3 & 7,5 & 0,54 & 0,10 & 0,93 & 15 & 1,1 & 2,5 \\
\hline Pouteria bangii (Rusby) T.D.Penn. & 4 & 10,0 & 0,72 & 0,04 & 0,40 & 20 & 1,4 & 2,5 \\
\hline Mangifera indica L. & 4 & 10,0 & 0,72 & 0,08 & 0,75 & 15 & 1,1 & 2,5 \\
\hline Artocarpus integrifólia Linn. f. & 1 & 2,5 & 0,18 & 0,22 & 1,95 & 5 & 0,4 & 2,5 \\
\hline Brosimum rubescens Taub. & 4 & 10,0 & 0,72 & 0,03 & 0,24 & 20 & 1,4 & 2,4 \\
\hline Rubiaceae & 1 & 2,5 & 0,18 & 0,19 & 1,70 & 5 & 0,4 & 2,2 \\
\hline Miconia hypoleuca (Benth.) Triana. & 4 & 10,0 & 0,72 & 0,05 & 0,42 & 15 & 1,1 & 2,2 \\
\hline Gustavia augusta L. & 3 & 7,5 & 0,54 & 0,05 & 0,46 & 15 & 1,1 & 2,1 \\
\hline Protium heptaphyllum (Aubl.) March. & 3 & 7,5 & 0,54 & 0,04 & 0,33 & 15 & 1,1 & 1,9 \\
\hline $\begin{array}{l}\text { Ocotea gardneri (Meisn.) Mez. } \\
\text { Eriotheca gracilipes (K.Schum.) A. }\end{array}$ & 3 & 7,5 & 0,54 & 0,04 & 0,32 & 15 & 1,1 & 1,9 \\
\hline Robyns. & 3 & 7,5 & 0,54 & 0,03 & 0,23 & 15 & 1,1 & 1,8 \\
\hline Plathymenia foliolosa Benth. & 2 & 5,0 & 0,36 & 0,08 & 0,69 & 10 & 0,7 & 1,8 \\
\hline NI 2 & 2 & 5,0 & 0,36 & 0,08 & 0,67 & 10 & 0,7 & 1,7 \\
\hline Myrtaceae 2 & 3 & 7,5 & 0,54 & 0,02 & 0,14 & 15 & 1,1 & 1,7 \\
\hline Clusia nemorosa G. Mey. & 3 & 7,5 & 0,54 & 0,05 & 0,48 & 10 & 0,7 & 1,7 \\
\hline Coиepia rufa Ducke. & 3 & 7,5 & 0,54 & 0,03 & 0,25 & 10 & 0,7 & 1,5 \\
\hline Nectandra cuspidata Nees \& Mart. & 3 & 7,5 & 0,54 & 0,03 & 0,23 & 10 & 0,7 & 1,5 \\
\hline Campomanesia sp. & 2 & 5,0 & 0,36 & 0,03 & 0,31 & 10 & 0,7 & 1,4 \\
\hline Sloanea sp. & 2 & 5,0 & 0,36 & 0,02 & 0,14 & 10 & 0,7 & 1,2 \\
\hline Genipa americana L. & 3 & 7,5 & 0,54 & 0,03 & 0,28 & 5 & 0,4 & 1,2 \\
\hline Erythroxylum squamatum Sw. & 2 & 5,0 & 0,36 & 0,01 & 0,10 & 10 & 0,7 & 1,2 \\
\hline NI 1 & 1 & 2,5 & 0,18 & 0,06 & 0,56 & 5 & 0,4 & 1,1 \\
\hline Chrysophyllum splendens L. & 2 & 5,0 & 0,36 & 0,04 & 0,36 & 5 & 0,4 & 1,1 \\
\hline Symphonia globulifera L.f. & 3 & 7,5 & 0,54 & 0,01 & 0,12 & 5 & 0,4 & 1,0 \\
\hline Luehea paniculata Mart. \& Zucc. & 2 & 5,0 & 0,36 & 0,03 & 0,29 & 5 & 0,4 & 1,0 \\
\hline $\begin{array}{l}\text { Coccoloba sp. } \\
\text { Guapira nítida (Mart. ex }\end{array}$ & 2 & 5,0 & 0,36 & 0,03 & 0,23 & 5 & 0,4 & 0,9 \\
\hline J.A.Schmidt) Lundell. & 1 & 2,5 & 0,18 & 0,02 & 0,15 & 5 & 0,4 & 0,7 \\
\hline
\end{tabular}




\begin{tabular}{lcccccccc}
\hline Ocotea sp. & 1 & 2,5 & 0,18 & 0,01 & 0,10 & 5 & 0,4 & 0,6 \\
Casearia arborea (Rich.) Urb. & 1 & 2,5 & 0,18 & 0,01 & 0,07 & 5 & 0,4 & 0,6 \\
Inga laurina (Sw.) Willd. & 1 & 2,5 & 0,18 & 0,01 & 0,05 & 5 & 0,4 & 0,6 \\
Myrtaceae 1 & 1 & 2,5 & 0,18 & 0,01 & 0,05 & 5 & 0,4 & 0,6 \\
Inga thibaudiana DC. & 1 & 2,5 & 0,18 & 0,00 & 0,04 & 5 & 0,4 & 0,6 \\
Annonaceae & 1 & 2,5 & 0,18 & 0,00 & 0,03 & 5 & 0,4 & 0,6 \\
TOTAL & 558 & 1395 & 100 & 11,2 & 100 & 1405 & 100 & 300 \\
\hline
\end{tabular}

*Onde: $\mathrm{N}=$ número de indivíduos amostrados na área de 0,4 ha; $\mathrm{DA}=$ Densidade Absoluta (indivíduos/ha); $\mathrm{DR}=$ Densidade Relativa (\%); DoA = Dominância Absoluta $\left(\mathrm{m}^{2} / \mathrm{ha}\right) ;$ DoR = Dominância Relativa $(\%) ; \mathrm{FA}=$ Frequência Absoluta (\%); FR = Frequência Relativa (\%); VI = Valor de Importância (\%).

Como representado na Figura 2, as espécies com maior valor de importância foram Tapirira guianensis, Schefflera morototoni, Pogonophora schomburgkiana, Cupania racemosa e Miconia prasina, respectivamente. A Tapirira guianensis e Schefflera morototoni, foram às únicas espécies que apresentaram em conjunto os maiores valores, respectivamente, de dominância, densidade e frequência. A espécie Pogonophora schomburgkiana apresentou bons valores de densidade e frequência dentro das parcelas, porém, como os diâmetros da espécie foram baixos, sua área basal também foi baixa.

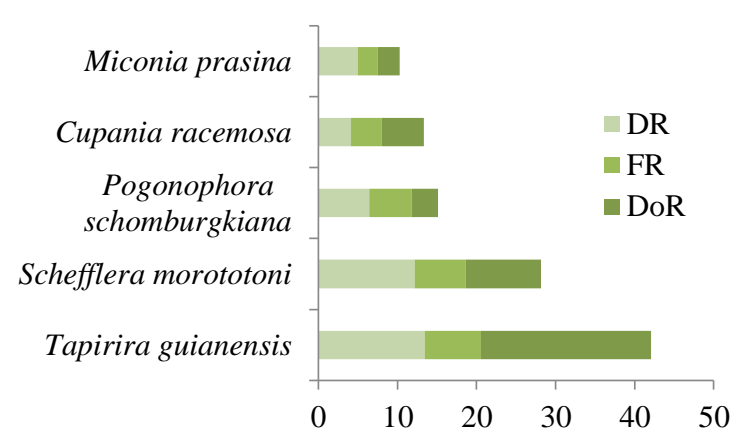

Figura 2 - Espécies com maior valor de importância estudados em um trecho da Mata do Ronca, Paulista - PE.

É possível afirmar que essas espécies possuem ampla distribuição em fragmentos de floresta atlântica localizados no Nordeste.

O Índice de Shannon foi de 3,44, comprovando que a diversidade florística da área amostrada está dentro dos padrões de riqueza encontrados em remanescentes florestais em Pernambuco, como os de Oliveira et al. (2012), no município de Moreno com 3,61 nats/ind; Rocha et al. (2008) apresentando 3,6 nats/ind em Igarassu e Silva Júnior et al (2008) apresentando 3,91 nats/indivíduo no Cabo de Santo Agostinho. Felfili e Rezende (2003) observaram que em ambientes florestais o valor da diversidade varia de 1,5 a 3,5 , podendo exceder 4 e alcançar em torno de 4,5 .

A Miconia prasina é uma espécie nativa pioneira, heliófita e pode ser um componente do sub-bosque de florestas primárias, ocorre em bordas e clareiras de fragmentos florestais (ANTONINI, FREITASNUNES, 2004). Conseguem se estabelecer rapidamente em solos degradados (SNOW, 1965; STILES, ROSSELLI, 1983).

Segundo Nunes, Somner e Dias (2010), a Cupania racemosa é uma espécie endêmica da Floresta Atlântica das regiões nordeste e sudeste e normalmente encontrada no interior desses remanescentes ou em pequenas clareiras.

A terceira espécie com maior valor de importância, Pogonophora schomburgkiana, é nativa e ocorre nas florestas atlânticas desde Pernambuco até o Espírito Santo. Por ser de rápido crescimento, é eficiente para o reflorestamento misto destinado a áreas de preservação (LORENZI, 2011).

De acordo com Ohashi e Leão (2005), a Schefflera morototoni é indicada para a recomposição de áreas alteradas. É uma espécie nativa e pode ser encontrada em florestas altas e densas de terra firme, capoeiras, capoeirões, margens de estradas e savanas. Por isso, possui classificações sucessionais desde espécie pioneira até clímax com exigência de luz. Porém, desenvolve-se melhor em floresta aberta, pouco densa e em vegetação secundária.

A Tapirira guianensis é uma espécie nativa amplamente distribuída no Brasil, ocorrendo em todos os estados, exceto no Rio Grande do Sul (COSTA, 2014). A espécie é altamente qualificada para reflorestamentos heterogêneos de áreas degradadas e de matas ciliares, também servindo como chamado para a fauna em geral por conta de seus frutos (LORENZI, 2002; SILVA-LUZ, 2011).

Mesmo dentro dos padrões de riqueza observados neste estudo, o Diagnóstico Socioambiental do Litoral Norte de Pernambuco (CPRH, 2003) afirma que esse fragmento florestal está em processo de degradação devido a expansão urbana. Problemas antrópicos como estradas, corte e queima de árvores, carros queimados e lixo também foram observados no local e colaboram com o baixo índice de preservação observado no trecho estudado o que pode ocasionar perda de biodiversidade e de processos ecológicos importantes para o funcionamento deste ecossistema.

A análise da síndrome de dispersão (Figura 3) demonstrou uma grande quantidade de espécies (51) 
dispersadas por animais (zoocoria), representando $80,9 \%$ do total. A outra forma de dispersão encontrada foi através de elementos abióticos (vento, água, etc.), com apenas 9 espécies sendo dispersadas dessa maneira no local $(14,3 \%)$. Esse padrão também foi verificado em fragmentos de floresta atlântica por Silva et al. (2012) e Oliveira et al. (2011). As florestas tropicais têm como característica apresentar altas proporções de espécies vegetais cuja dispersão é feita por animais (zoocoria), com cerca de 70 a $90 \%$ das espécies (MORELLATO, LEITÃO-FILHO, 1992; TABARELLI, PERES, 2002), confirmando os resultados do presente estudo.

\section{Síndrome de dispersão das espécies de um trecho da Mata do Ronca - PE}

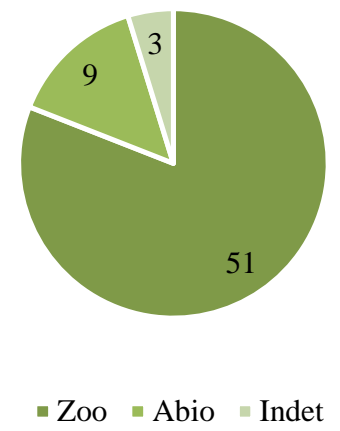

Figura 3 - Síndrome de dispersão das espécies de um trecho da Mata do Ronca, Paulista - PE.

\section{CONCLUSÕES}

Apesar das interferências antrópicas do local, a comunidade estudada, com base no levantamento fitossociológico, mostra que o componente arbóreo adulto se encontra dentro dos padrões de riqueza e abundância encontrados em remanescentes florestais de Pernambuco.

As espécies mais importantes do trecho foram, respectivamente, Tapirira guianensis, Schefflera morototoni, Pogonophora schomburgkiana, Cupania racemosa e Miconia prasina. Estas espécies possuem ampla distribuição em fragmentos de floresta atlântica localizadas no Nordeste.

Em relação a síndrome de dispersão foi observado que $84,7 \%$ possuem a dispersão de sementes feita por animais colaborando com o fato de a dispersão zoocórica ser a mais importante para as florestas tropicais.

\section{REFERÊNCIAS BIBLIOGRÁFICAS}

ANTONINI, R. D.; FREITAS-NUNES, A. F.; Estrutura populacional e distribuição espacial de Miconia prasina D.C. (Melastomataceae) em duas áreas de Floresta Atlântica na Ilha Grande, RJ, Sudeste do Brasil. Acta Botânica Brasílica. 18(3): 671-676. 2004.
APG III. 2009. An update of the Angiosperm phylogeny Group classification for the orders and families of flowering plants: APG III. Botanical Journal of the Linnean Society, 161: 105-121.

BULHÕES, A. A. de. et al. Levantamento florístico e fitossociológico das espécies arbóreas do bioma Caatinga realizado na Fazenda Várzea da Fé no Município de Pombal-PB. Informativo Técnico do Semiárido, Pombal, v. 9, n. 1, p. 51-56, jan/jun. 2015.

BUSTAMANTE, R. O.; CANALS, M. 1995. Dispersal quality in plants: How to measure efficiency and effectiveness of a seed disperser. Oikos 73 (1): 133-136. CHAVES, A. D. C. G., SANTOS, R. M. D. S., SANTOS, J. O. D., FERNANDES, A. D. A., \& MARACAJÁ, P. B. (2013). A importância dos levantamentos florístico e fitossociológico para a conservação e preservação das florestas. Agropecuária Científica No Semiarido, 9(2), 43-48.

COMPANHIA PERNAMBUCANA DO MEIO AMBIENTE - CPRH. Diagnóstico socioambiental do litoral norte de Pernambuco. Recife: 2003. 214p.

COSTA, C. C. et al. Conhecendo Espécies de Plantas da Amazônia: Tatapiririca (Tapirira guianensis Aubl. - Anacardiaceae). Concórdia: Embrapa Amazônia Oriental, 2014. 6 p. (Embrapa Amazônia Oriental. Comunicado Técnico, 253).

FELFILI. J. M.; REZENDE. R. P. Conceitos e Métodos em Fitossociologia. Brasília: UNB. Departamento de Engenharia Florestal. 2003. 68 p.

FREITAS, W. K.; MAGALHÃES, L. M. S. 2012. Métodos e Parâmetros para Estudo da Vegetação com Ênfase no Estrato Arbóreo. Floresta e Ambiente 2012 out./dez.; 19(4): 520-540.

HOWE, H. F.; J. SMALLWOOD. 1982. Ecology of seed dispersal. Annual Review of Ecology and Systematics 13: 201-228.

LORENZI, H. Árvores brasileiras: manual de identificação e cultivo de novas plantas arbóreas nativas do Brasil. Nova Odessa: Instituto Plantarum, 2002. v. 1, $368 \mathrm{p}$.

LORENZI, H. Árvores brasileiras: manual de identificação e cultivo de novas plantas arbóreas nativas do Brasil. Nova Odessa: Instituto Plantarum, 2011. v. 3, $385 \mathrm{p}$.

MARTINS, F. R. Estrutura de uma floresta mesófila. Campinas: Ed. UNICAMP; 1991.

MORELLATO, L.P.C.; LEITÃO-FILHO, H.F. 1992. Padrões de frutificação e dispersão na Serra do Japi, p. 112-141. In: Morellato, L.P.C. (Org.) História Natural da Serra do Japi: ecologia e preservação de uma área florestal no Sudeste do Brasil. Editora da UNICAMP/FAPESP, Campinas. 
NUNES, A. F.; SOMNER, G. V.; DIAS, A. O. Cupania racemosa (Vell.) Radlk. (Sapindaceae) e a entomofauna associada em um fragmento de Mata Atlântica, Instituto Zoobotânico de Morro Azul, Engenheiro Paulo de Frontin, RJ, Brasil. In: $61^{\circ}$ Congresso Nacional de Botânica, 2010. Manaus. Anais... Disponível em: < http://www.botanica.org.br/trabalhos-

cientificos/61CNBot/ResumoS02_CNBot_2010_1028.pdf

.>. Acesso em: 05 de dezembro de 2018 .

OHASHI, S. T.; LEÃO, N. V. M. Morototó - Schefflera morototoni (Aubl.) Maguire, Steyerm. \& Frodin. Concórdia: Informativo Técnico Rede de Sementes da Amazônia, 2005. 2 p. (Informativo Técnico Rede de Sementes da Amazônia, No 12).

OLIVEIRA, S., OEHLER, F., SAN-MIGUELAYANZ, J., CAMIA, A., PEREIRA, J. M. C. (2012). Modeling spatial patterns of fire occurrence in Mediterranean Europe using Multiple Regression and Random Forest. Forest Ecology and Management, 275, 117-129.

OLIVEIRA, L. S. B.; MARAGON, L. C.; FELICIANO, A. L. P.; DE LIMA, A. S.; CARDOSO, M. O.; DA SILVA, V. F. Florística, classificação sucessional e síndromes de dispersão em um remanescente de Floresta Atlântica, Moreno, PE. Revista Brasileira de Ciências Agrárias, Recife, v. 6, n. 3, p. 502-507, 2011.

PEDRONI, F. 1993. Ecologia de copaíba (Copaifera langsdorffii Desf. Caesalpinaceae) na Reserva Municaipal de Santa Genebra. Dissertação de Mestrado, Universidade Estadual de Campinas, Campinas, SP.

ROCHA, K. D.; CHAVES, L. F. C., MARANGON, L. C.; LINS E SILVA, A. C. B. Caracterização da vegetação arbórea adulta em um fragmento de floresta atlântica, Igarassu, PE. Revista Brasileira de Ciências Agrárias, 2008, 3 (Janeiro-Março). Disponível em: <http://www.redalyc.org/articulo.oa?id=119017261007>. Acesso em: 27 de novembro de 2018.
SILVA JÚNIOR, F. et al. Fitossociologia do componente arbóreo em um remanescente de Floresta Atlântica no Município do Cabo de Santo Agostinho, PE. Revista Brasileira de Ciências Agrárias, 2008, 3 (JulhoSetembro). Disponível em: https://www.redalyc.org/comocitar.oa?id=119017386013 >. Acesso em: 27 de novembro de 2018.

SILVA-LUZ, C. L. Anacardiaceae R. Br. na flora fanerogâmica do Estado de São Paulo. 2011. $94 \mathrm{f}$. Dissertação (Mestrado em Botânica) - Programa de Pósgraduação em Botânica IBUSP, Universidade do Estado de São Paulo, São Paulo.

SILVA, R. K. S. da; FELICIANO, A. L. P.; MARAGON, L. C.; LIMA, R. B. A.; SANTOS, W. B. dos. Estrutura e síndromes de dispersão de espécies arbóreas em um trecho de mata ciliar, Sirinhaém, Pernambuco, Brasil. Pesquisa Florestal Brasileira, Colombo, v. 32, n. 69, p. 1-11, jan./mar. 2012.

SNOW, D.W. 1965. A possible selective factor in the evolution of fruiting seasons in Tropical Forests. Oikos 15: 274-281.

STILES, F.G. \& ROSSELLI, L. 1983. Consumption of fruits of the Melastomataceae by birds: how diffuse is coevolution? Vegetatio 107/108: 57-75.

TABARELLI, M.; PERES, C.A. 2002. Abiotic and vertebrate seed dispersal in the Brazilian Atlantic forest: implications for forest regeneration. Biological Conservation, 106: 165-176.

VAN DER PIJL, L. 1982. Principles of dispersal in higher plants. 3rd ed. Springer-Verlag, Berlin. 